\title{
Les municipalités françaises face à la gestion des ordures ménagères : difficultés de la mise en place d'un nouveau système
}

French Town Councils facing problems of garbage management: The difficulties of setting up a new system

Die Entsorgung des Haushaltmülls in den französischen Gemeinden;

Schwierigkeiten der Einführung eines neuen Systems

\section{Estelle Kah}

\section{(2) OpenEdition}

\section{Journals}

Édition électronique

URL : http://journals.openedition.org/rge/4466

DOI : $10.4000 /$ rge.4466

ISSN : $2108-6478$

Éditeur

Association des géographes de l'Est

Édition imprimée

Date de publication : 1 avril 1999

ISSN : 0035-3213

\section{Référence électronique}

Estelle Kah, « Les municipalités françaises face à la gestion des ordures ménagères : difficultés de la mise en place d'un nouveau système », Revue Géographique de l'Est [En ligne], vol. 39 / 2-3 | 1999, mis en ligne le 05 septembre 2013, consulté le 08 septembre 2020. URL : http://journals.openedition.org/ rge/4466; DOI : https://doi.org/10.4000/rge.4466

Ce document a été généré automatiquement le 8 septembre 2020

Tous droits réservés 


\title{
Les municipalités françaises face à la gestion des ordures ménagères : difficultés de la mise en place d'un nouveau système
}

\author{
French Town Councils facing problems of garbage management: The difficulties \\ of setting up a new system \\ Die Entsorgung des Haushaltmülls in den französischen Gemeinden ; \\ Schwierigkeiten der Einführung eines neuen Systems
}

Estelle Kah

1 Le déchet, l'objet ou le résidu que l'on jette quotidiennement, est le résultat inévitable de tout cycle de vie d'un produit. Mais qu'est ce qu'un déchet? Qu'elles sont les représentations, les pratiques et les enjeux qui lui sont associés ? L'histoire des déchets est révélatrice de la capacité de l'homme à faire preuve d'ingéniosité pour se débarrasser de ses rebuts. Il a su imaginer les moyens de confier à la nature le soin d'assimiler ses restes en pratiquant l'enfouissement, le compostage, le brûlage ou en les donnant en pâture aux animaux domestiques. Cette histoire du déchet s'inscrit dans une dynamique complexe des changements de valeur et, selon G. Bertolini (1990) «le déchet est, sinon miroir, du moins reflet de société; il en reflète la culture et les valeurs ». En France, elle met en évidence la prise de conscience tardive pour élaborer et mettre en place une véritable politique de gestion des déchets urbains ${ }^{1}$. Le service d'élimination des déchets urbains est assurée juridiquement par les communes depuis la loi du 15 juillet 1975. Cette loi est actuellement remise en cause. En effet, l'évolution majeure du cadre législatif et réglementaire de 1992, avec la loi-cadre du 13 juillet sur la gestion des déchets ménagers et assimilés et le décret du premier avril relatif aux déchets d'emballage, devrait être l'élément déclencheur de la réorganisation des schémas traditionnels de gestion. Le temps est révolu où l'on donnait à choisir aux communes entre l'incinération, le compostage ou la décharge. Cette loi indique qu'il sera impossible d'ici l'an 2002 de mettre en décharge des déchets urbains et plus 
particulièrement les ordures ménagères $(\mathrm{OM})^{2}$ qui n'auront pas au préalable été valorisées. Elle prévoit que seuls les déchets ultimes pourront être éliminés en $\mathrm{CET}^{3}$, les autres devant être obligatoirement valorisés. Les formes de valorisation sont au nombre de trois (valorisation «matière " par le recyclage, valorisation organique par le compostage, valorisation énergétique ou thermique avec récupération d'énergie).

2 La prévention quantitative et qualitative, ainsi que la valorisation sont prioritaires par rapport à l'élimination simple. La collecte, qui semblait un acquis, redevient un enjeu important, car le tri, ou le non mélange à la source, impose un service de ramassage diversifié selon les catégories de déchets et les types de producteurs. Conséquence logique, le traitement lui-même devient multimodes. La conception du déchet est donc liée à l'idéologie qu'on lui associait : immondice, résidu, rebut ou ordure, il a actuellement un caractère multiacteurs, multiarguments, tandis que le gisement des déchets ménagers est lui multimatériaux. Les municipalités doivent maintenant quitter une vision unitaire de l'élimination des déchets pour s'engager dans l'élaboration d'un système de gestion séparative des ordures ménagères. Mais en ont-elles les capacités?

\section{Des charges nouvelles pour les communes}

3 Les préoccupations essentielles des communes vis-à-vis des déchets ménagers sont liées à leur croissance, leur diversification et aux moyens traditionnels d'élimination qui sont insuffisants et polluants. En outre, le cadre juridique renouvelé récemment implique de réorganiser les schémas classiques d'élimination pour se diriger vers une gestion de valorisation, séparative et multiforme des ordures ménagères. La situation actuelle peut être résumée par quelques statistiques.

\section{A. Croissance et diversification des déchets ménagers}

4 En France, depuis un siècle, l'élévation du niveau de vie et des modes de consommation a provoqué un accroissement considérable du volume des déchets ménagers. Les données disponibles à ce sujet sont globalement très partielles et insuffisantes. Aucune statistique n'est utilisable pour mesurer l'augmentation des déchets ménagers sur cette période ; mais il existe des chiffres pour certaines agglomérations qui précisent cette croissance. Ainsi, le tonnage collecté à Paris s'est accru de près de $50 \%$ de 1922 à 1984, alors que dans le même temps la population diminuait de $25 \%$ : il est passé de $0,7 \mathrm{~kg}$ par jour et par habitant à 1,3 kg. Le rapport du GEERS ${ }^{4}$ en 1974 évaluait la production des ordures ménagères pour l'année 1972 à 11 millions de tonnes avec une production annuelle par habitant de $290 \mathrm{~kg}$ en moyenne. En 1960, elle était de $220 \mathrm{~kg}$ par habitant ; elle est de $358 \mathrm{~kg}$ en 1990, soit par jour et par habitant environ 0,98 $\mathrm{kg}$ de déchets ménagers (M. Pelchat, 1993). Les 55 millions de français ont généré en 1990, 30 millions de tonnes de déchets ménagers dont 20 millions d'ordures ménagères, soit $360 \mathrm{~kg}$ par habitant. En 1991, l'ANRED ${ }^{5}$ estimait la production d'ordures ménagères à 20,5 millions de tonnes et la quantité par habitant à $385 \mathrm{~kg} / \mathrm{an}$, il ne s'agit là que d'une moyenne nationale. De plus, selon les projections du CREDOC ${ }^{6}$, il était prévu que les Français jetteraient 1,2 kg de déchet par jour en 1995, soit $450 \mathrm{~kg}$ par habitant et par an (M. Pelchat, 1993). Or, la quantité de déchets ménagers générée, actuellement utilisée comme référence de base dans de nombreuses circonstances, tourne autour de la valeur remarquable de $1 \mathrm{~kg} / \mathrm{hab} /$ jour, soit $365 \mathrm{~kg} / \mathrm{hab} / \mathrm{an}$, (Ademe ${ }^{7}, 1994$ (a)). Pour autant, ce 
ratio est utilisé de façon uniforme sur tout le territoire, bien qu'il ne représente qu'une moyenne nationale et ne tienne pas compte des disparités locales.

Non seulement ces déchets ménagers augmentent dans des proportions variées selon les lieux de production (zones rurales ou urbaines...), mais en plus ils se diversifient. En effet, jusqu'à présent les déchets ménagers se définissaient par leur caractère non dangereux. Ils ne devaient pas être générateur de nuisance par opposition à d'autres déchets dits à risques. Or, force est de constater que de nouveaux déchets apparaissent, et qu'un certain nombre d'ordures ménagères rejetées quotidiennement sont toxiques ; elles ne peuvent donc plus être traitées comme des déchets inertes. De ce fait, l'évolution des risques liés aux déchets ménagers, associée à l'augmentation de leurs flux mal identifiés, amène certains acteurs à reconsidérer leurs points de vue.

\section{B. Les nouvelles dispositions réglementaires}

6 L'élimination, qui regroupe la fonction de collecte et de traitement des déchets urbains, est un service public communal. Le service d'élimination des ordures ménagères s'est structuré à partir des années 1970. Il a évolué progressivement de l'activité de simple collecte à l'activité de collecte et de traitement durant la décennie suivante. Il se dirige maintenant vers une spécialisation des traitements et des techniques par l'obligation faite aux municipalités de valoriser les déchets ménagers. «Ce service est actuellement multiforme et il subit aujourd'hui une mutation qui l'amènera à une véritable logique industrielle» (A. Le Bozec, 1994). Les principaux éléments responsables de cette mutation sont liés aux considérations environnementales, économiques, politiques, aux attentes des usagers en matière de qualité et de protection de l'environnement et à l'évolution des nouvelles contraintes réglementaires en la matière.

\section{- Des attentes nouvelles en matière de qualité de vie et d'environnement}

7 «À partir des années 1985, le service de gestion des déchets ménagers fonctionne dans la totalité des communes, mais les attentes des usagers se tournent vers de nouvelles préoccupations et exigences: la qualité des prestations et une meilleure prise en compte de l'environnement » (Malhuret C., cité par Defeuilley C., 1996). Cela se traduit notamment par la contestation des modes d'organisation et des techniques utilisées. La gestion des déchets ménagers fondée essentiellement sur l'élimination - mise en décharge et incinération sans récupération (Fig. 1) - se heurte à des blocages sociaux concernant l'implantation de nouvelles unités de traitement notamment. Ce phénomène s'exprime par le syndrome $\mathrm{NIMBY}^{8}$. Le système unique d'élimination qui engendre des pollutions et du gaspillage est remis en cause. Les municipalités ne peuvent donc plus se contenter de répondre à la demande de manière uniquement quantitative. La connaissance des flux de déchets (tonnages) qui compose le gisement de déchets municipaux, et plus particulièrement des déchets ménagers, est fondamentale pour une valorisation maximale.

8 La figure 1 résume succinctement la situation de la gestion des déchets municipaux fondée essentiellement sur l'élimination simple. La globalité du gisement des déchets municipaux est collectée sans différenciation des producteurs, et éliminée sans valorisation. 
Figure 1 : Circuit d'élimination des déchets municipaux au début des années 70

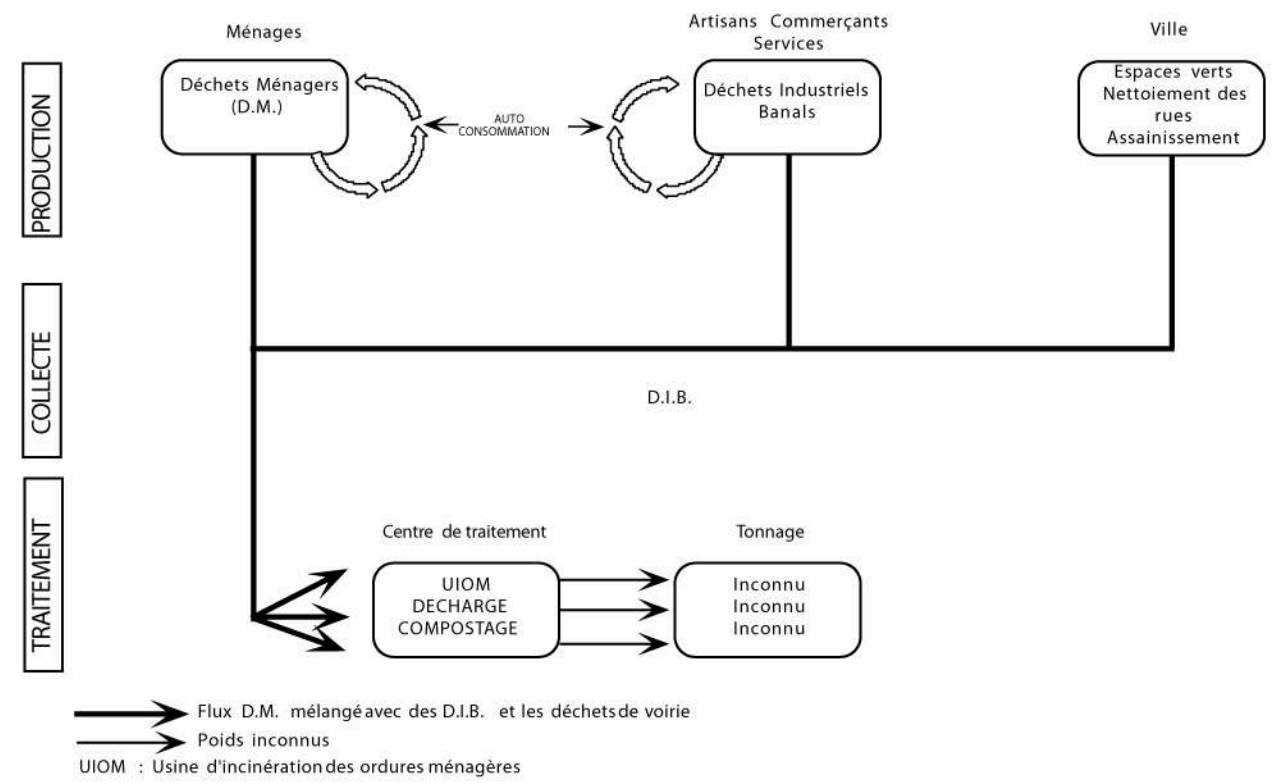

- Les répercussions sur le rôle des municipalités : des contraintes réglementaires et techniques

9 Les nouvelles contraintes réglementaires et techniques sont une réponse des politiques aux exigences des usagers. Le traitement doit désormais respecter des normes précises, et la réglementation impose le développement de schémas complexes, organisés autour d'une utilisation complémentaire des techniques (fig. 2). La loi de 1992 est l'élément déclencheur de la réorganisation des schémas traditionnels de gestion. Elle relance la planification de la gestion des déchets en demandant aux préfets d'établir un plan départemental ou interdépartemental pour coordonner et orienter l'ensemble des actions nécessaires à la réalisation des objectifs définis par la loi, à savoir la prévention, la valorisation et le stockage limité aux déchets ultimes. Les principales innovations que cette loi introduit sont les suivantes. 
Figure 2 : Circuit prévisionnel de valorisation des déchets ménagers et assimilés à l'horizon 2002

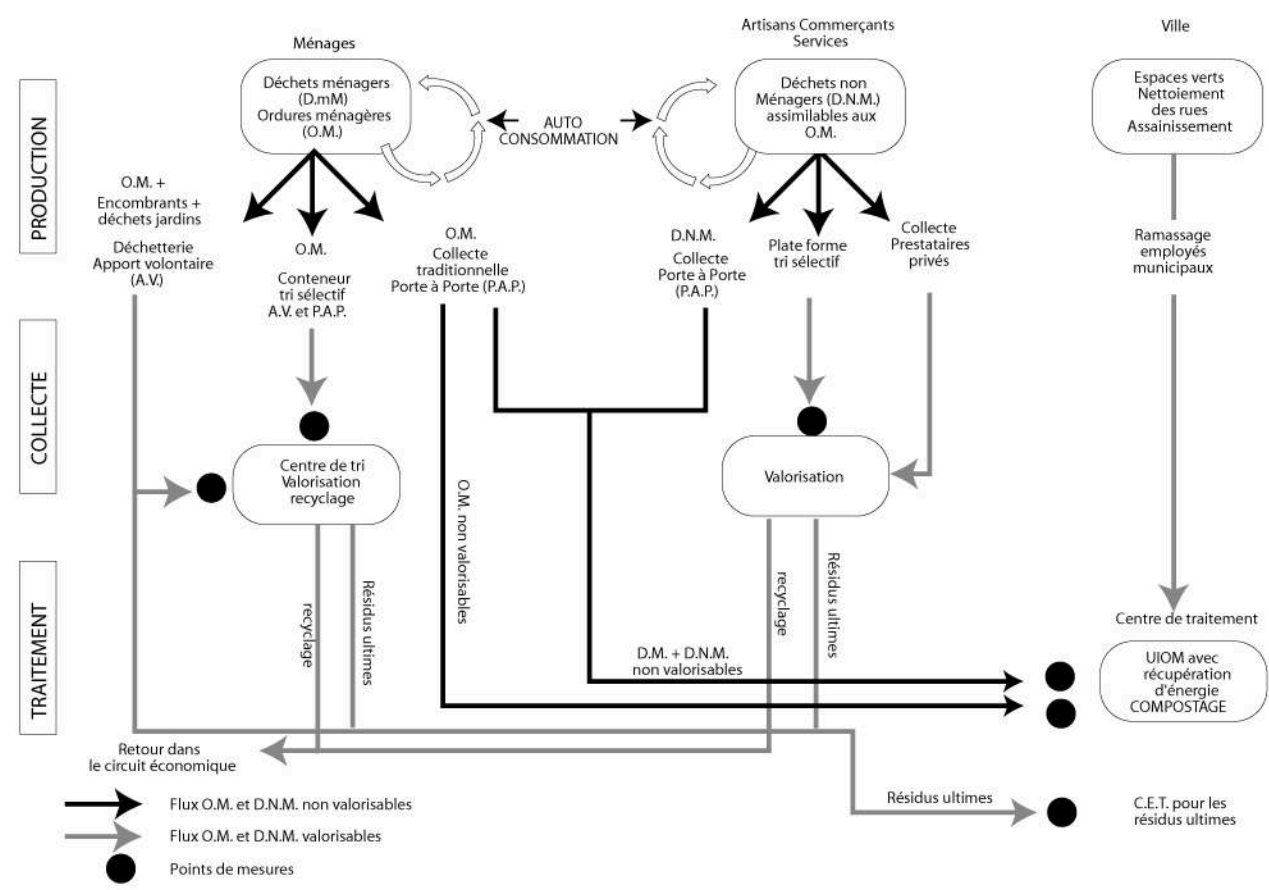

10 Cette figure, représente la situation à atteindre au sens de loi de 1992 d'ici 2002. Les flux de déchets et les types de producteurs sont distinctement identifiés. Les filières de valorisation sont adaptées aux différents flux de déchets et les résidus ultimes sont stockés en CET.

11 - une réorganisation des procédés d'élimination : il devrait être impossible d'ici l'an 2002 de mettre en décharge des déchets n'ayant pas été au préalable valorisés, soit par recyclage, soit par incinération avec récupération d'énergie. Il n'y aura à terme qu'une seule catégorie de décharges n'acceptant que les déchets ultimes (fig. 2).

12 - une nouvelle forme de financement pour contribuer à la prise en charge de cette politique de modernisation : une taxe évolutive est versée au profit d'un fond de modernisation de la gestion des déchets ménagers (FMGD) ${ }^{9}$ géré par l'ADEME et ce, pour aider à la réalisation d'installations de traitement. La loi Barnier, votée le 19 janvier 1995, a fixé le montant de 20 francs par tonne en 1996, 30 francs pour 1997 et 40 francs pour 1998. La loi précise également, que la redevance spéciale prévue pour les déchets non ménagers (DNM) ${ }^{10}$ assimilables aux déchets ménagers dont les communes assurent l'élimination, doit être instaurée.

13 - une nouvelle répartition des tâches entre les communes et leurs groupements : la loi impose l'établissement, sous l'autorité du préfet, de plans départementaux pour les déchets ménagers, qui décrivent les filières de traitement existantes et celles qui sont à mettre en place. Ils permettent de mettre en pratique le principe de proximité selon lequel les déchets produits dans l'espace départemental ou interdépartemental doivent y être traités. Ces plans visent à limiter le plus possible le transport des déchets ménagers et assimilés entre les départements (ADEME 1993, (c)).

14 Le dispositif est complété par le décret du 1er avril 1992 relatif aux déchets d'emballage des ménages qui prévoit que tout producteur ou importateur de produits commercialisés dans des emballages est tenu de contribuer ou de pourvoir à l'élimination de l'ensemble de ses déchets d'emballage issus des ordures ménagères. Le 
décret a ainsi pour objectif de rendre les industriels responsables de l'élimination des déchets d'emballage mis sur le marché : soit ils se chargent eux-mêmes de les récupérer (mais il apparaît difficile que chaque industriel les récupère auprès des ménages), soit ils font appel à des organismes collectifs agréés par les pouvoirs publics. Pour l'essentiel des emballages ménagers, le financement sera probablement assuré par une redevance versée à un organisme agrée choisi par les industriels. Ces organismes contribuent, avec les collectivités locales, au développement des collectes sélectives et des tris de déchets d'emballage. Ce sont donc les collectivités locales qui seront concernées par le tri et le recyclage. Le passage obligé à la valorisation s'accompagne aussi d'évolutions techniques et surtout organisationnelles. Avec ces nouvelles réglementations, les municipalités doivent passer d'une gestion relativement simple des techniques traditionnelles de collecte/traitement (fig. 1 et fig. 3) à des configurations complexes, organisés autour d'ensembles techniques complémentaires (fig. 2).

Figure 3 : Circuit de collecte de traitement des déchets ménagers et assimilés depuis 1992

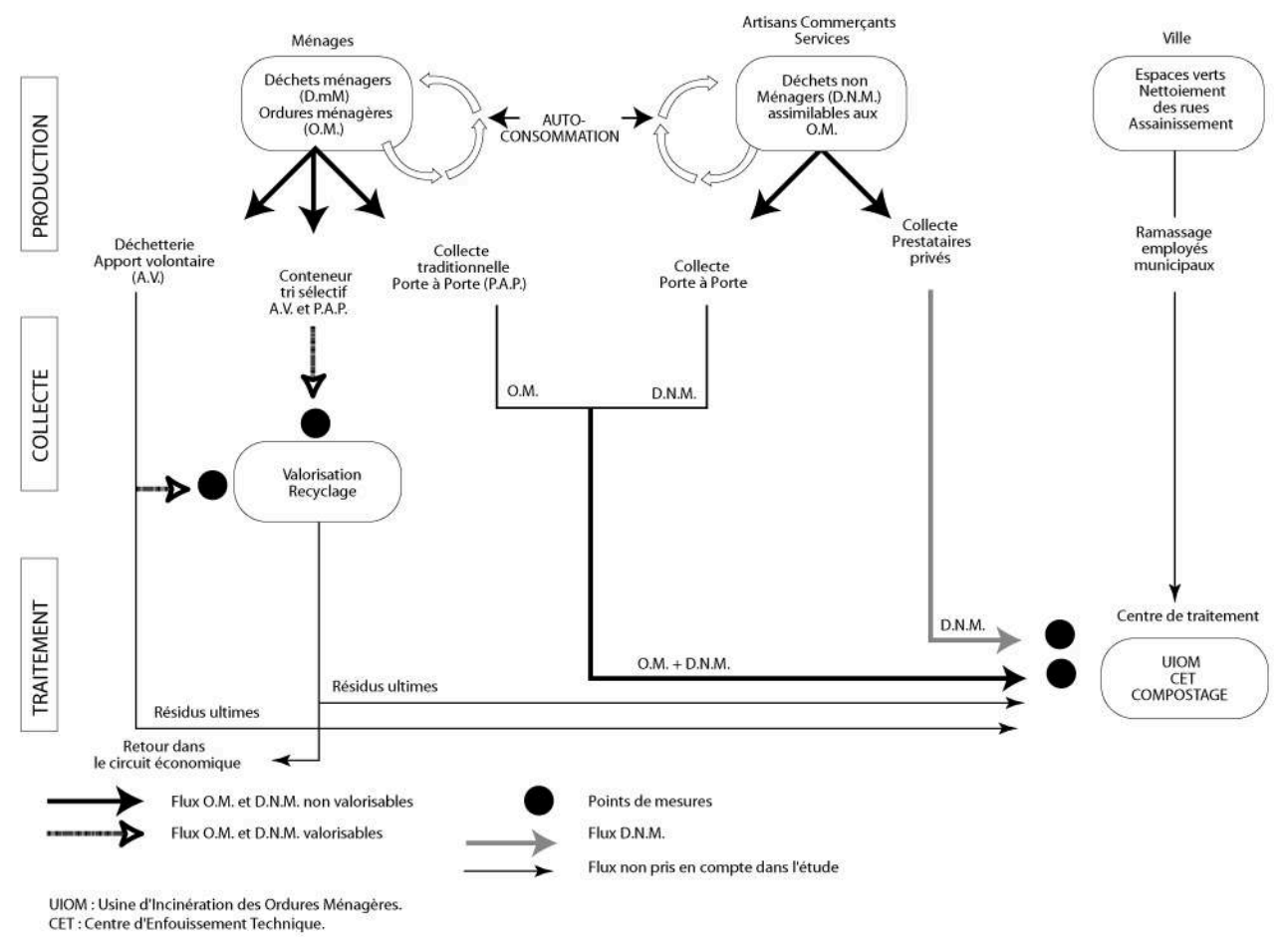

\section{Conséquences pour les municipalités}

Ces évolutions réglementaires se traduisent par une augmentation des difficultés pour assurer l'exploitation technique et financière des nouvelles formes d'organisation des schémas de collecte et de traitement des déchets ménagers. Les origines de ces tâches, plus difficiles à maîtriser, sont la technicité accrue des équipements et le choix des filières de valorisation à adopter. 


\section{- Accroissement de la technicité des équipements}

16 acteurs car les compétences requises sont plus importantes pour faire fonctionner les équipements de traitement. Par ailleurs, l'exploitation de ces installations doit faire preuve de plus de professionnalisme pour respecter et suivre les nouvelles normes, sans avoir à supporter des coûts trop importants.

\section{- Des schémas d'organisation plus complexes à mettre en place}

À mesure que les réglementations et les techniques s'additionnent, l'organisation du service devient plus difficile à mettre en place. Il s'agit pour les municipalités de réorganiser les schémas traditionnels de collecte/traitement vers des schémas fondés sur le fonctionnement simultané de diverses techniques tout en y faisant participer les usagers (fig. 2) : les techniques de pré-collecte, de collecte (traditionnelle et sélective) et de traitements sont interdépendantes. Le choix d'une pré-collecte influe sur les qualités de collecte et de tri qui vont conditionner l'efficacité de valorisation et donc de traitement des déchets ménagers. De plus, il faut une cohérence entre les choix de collecte envisagés et les possibilités locales de traitement existantes. Les éléments essentiels pour atteindre ces objectifs sont la participation des usagers aux tris, ainsi que la connaissance quantitative et qualitative du gisement considéré. Une municipalité mettant en place des collectes sélectives sans connaître la part de participation des usagers et le tonnage d'ordures qu'elle est censée collecter se trouvera dans une situation délicate à gérer.

Cette figure caractérise l'état actuel et transitoire de l'organisation de la gestion des déchets municipaux. Des collectes sélectives s'ajoutent aux collectes traditionnelles. Les producteurs de DNM sont distingués des producteurs ménages et mettent en place des filières de valorisation interne. De nouveaux centres de traitements sont intégrés dans le circuit, notamment des centres de tri et des UIOM $^{11}$ avec récupération d'énergie. Les décharges sont fermées ou remplacées par des CET réservés aux résidus ultimes. Les flux de déchets collectés commencent à être systématiquement pesés.

\section{- Élévation des coûts de collecte et de traitement des ordures ménagères}

Les normes de 1986 et de 1991 ont provoqué une augmentation des coûts d'exploitation et d'investissement, qui passent de $165 \mathrm{~F} / \mathrm{t}$ en moyenne vers le milieu des années 1980, à environ $350 \mathrm{~F} / \mathrm{t}$ au début des années 1990 (ADEME, 1993 (a)). Tous ces prix sont indicatifs et sont soumis à de nombreux facteurs de variation, notamment locaux (ADEME, 1993 (b)). La loi de 1992, qui prévoit l'usage des décharges uniquement pour les déchets ultimes d'ici 2002, se traduit par une généralisation des collectes sélectives et un développement des équipements de valorisation, incinération avec récupération d'énergie, compostage et méthanisation, centre de tri et déchetteries. Le respect des nouvelles réglementations devrait entrainer, selon les projections de l'ADEME, la construction de 160 usines d'incinération, de 200 équipements de tri et de diverses autres unités (compostage, centre de transfert, déchetteries). Ces techniques sont plus coûteuses que la simple collecte $(900 \mathrm{~F} / \mathrm{t}$ contre $350 \mathrm{~F} / \mathrm{t})$, et vont donc peser de manière importante sur le coût global de la gestion des déchets ménagers. 


\section{Les nouveaux besoins des communes pour répondre à ces mutations} l'époque de la filière unique, l'élimination des déchets ménagers faisait appel à une seule technique : ils étaient collectés en mélange et dirigées vers une décharge ou un incinérateur (fig. 1). Actuellement, les multiples techniques de traitement sont considérées comme complémentaires les unes des autres. Dans la mesure où elles s'appliquent à des fractions différentes des ordures ménagères, la collecte et le tri deviennent des phases clés de la gestion des déchets (fig. 2). Autrement dit, pour atteindre ces objectifs, les communes doivent réussir à mettre en place des collectes sélectives, inciter les usagers à trier, instaurer la redevance spéciale pour les DNM, et établir des facturations selon le service rendu (fig. 3). Actuellement, les ordures n'étant pas systématiquement pesées, les municipalités ne disposent que d'une approximation des quantités qu'elles peuvent valoriser et a fortiori facturer. La question du financement de la collecte et du traitement des déchets est donc très complexe et l'idée d'une facturation fine bien difficile à mettre en place dans la mesure ou il faut tenir compte des poids, des volumes, des récupérations possibles ou existantes, de la solvabilité des producteurs etc. !... Des indicateurs de production spécifique sont donc nécessaires pour évaluer les quantités d'ordures ménagères collectées soit globalement à l'échelle de la commune, soit individuellement selon le type de producteur considéré (ménage, entreprise).

\section{Vers une évaluation de la production des ordures ménagères}

21 Jusqu'au début des années 1990, les gestionnaires du déchet ne se souciaient que partiellement de la quantité des déchets produits et traités sur un espace géographique délimité. Aujourd'hui, les communes ne connaissent pas les tonnages générés sur leur territoire : elles collectent des "masses de déchets ». Elles ne sont pas en mesure de distinguer la part de ce qui est généré par les différents producteurs de déchets, notamment les ordures ménagères, des déchets assimilés aussi appelés DNM. De même, les niveaux d'organisation technique qu'elles mettent en place, collecte et centres de traitement, sont uniquement appréhendés par la masse ou le volume de déchets potentiellement générés sur leur territoire. La fonction technique du service propreté d'une commune consiste donc uniquement à répondre à une prise en charge du gisement de déchets sur leur territoire, sans trop différencier les catégories et les producteurs de déchets. Or, la connaissance des flux de déchets, que ce soient les ordures ménagères, les déchets non ménagers ou autres déchets de la commune, est fondamentale pour la mise en place d'une gestion optimale de valorisation des déchets. Comme il n'existe pas encore de système de pesée systématique et généralisable sur tout le territoire national, les gestionnaires se fondent sur l'indicateur d'approche des besoins : le kilo par habitant et par an, utiliser de façon uniforme sur tout le territoire, mais qui a montré ses limites tel qu'il a été calculé et utilisé jusqu'à présent.

Revue Géographique de l'Est, vol. 39 / 2-3 | 1999 


\section{A. Difficultés pour mesurer les ordures ménagères dans chaque commune} citées proviennent de l'ADEME, et, en particulier, de l'observatoire National des Déchets situé à Angers. Outre le problème posé par l'insuffisance de données, la terminologie en matière de déchet et notamment des déchets ménagers est une difficulté supplémentaire. L'appellation déchets ménagers, utilisée par les acteurs en la matière, recouvre en fait plusieurs types de déchets. Elle provient de la définition issue de la loi de 1975, qui distingue les déchets des ménages, premier type de producteurs qui relève des communes, et les déchets industriels à risque, deuxième type, qui relève des entreprises. Les communes sont donc, pour leur part, tenues d'assurer l'élimination des déchets que l'on peut répartir suivant leur origine en catégories: les déchets ménagers, les déchets d'origine commerciale ou artisanale (DIB) ${ }^{12}$ et les déchets de voirie... Ces déchets sont dits municipaux ou urbains parce qu'ils apparaissent sur le territoire communal et qu'ils ne sont pas liés aux processus industriels de fabrication. Par ailleurs, certains types de producteurs sont mal identifiés. Les déchets de la petite industrie, de l'artisanat et du commerce sont mal pris en compte. Sur le fond, ils ne sont ni des déchets ménagers, ni des déchets de grosses industries ou d'industries à risque. Pourtant, ils suivent souvent les mêmes voies d'élimination que les déchets ménagers puisqu'ils y sont assimilés. Ces confusions sont une source d'erreurs notables qui peuvent remettre en cause les résultats des tonnages dits " d'ordures ménagères ». Il est à l'heure actuelle encore extrêmement difficile de quantifier la part des OM des DNM, ou des DIB. Il n'existe donc pas de système d'évaluation qui permette une bonne caractérisation des déchets urbains. Les difficultés auxquelles sont confrontées les communes pour obtenir des tonnages exhaustifs et précis sont donc de plusieurs ordres

- Il n'existe pas de système de pesée homogène que ce soit au niveau des poubelles des usagers, des camions de collecte ou des centres de traitement. Quand des pontsbascules existent à l'entrée de certains centres de traitement, les déchets pesés sont mélangés, ils ne sont pas assez représentatifs, pour permettre une généralisation, par extrapolation des résultats, au niveau national.

24 Les modes de gestion - collecte et traitement - sont variés et très différents d'une commune à l'autre, d'un $\mathrm{EPCI}^{13}$ à un autre. Il y a régulièrement mélange entre DNM et les OM (cf. fig. 4).

25 - Concernant les ordures ménagères, il est peu aisé d'évaluer par commune la part d'ordures ménagères collectée traditionnellement des ordures ménagères collectées séparément par tri sélectif (apport volontaire, porte à porte ou en déchetterie).

- Pour les DNM, il est complexe de distinguer avec exactitude, quelles sont les entreprises qui font partie de la collecte traditionnelle par rapport à celles qui effectuent des collectes séparatives (circuit de collecte distinct de la collecte traditionnelle), ou du tri sélectif interne.

27 - Les points de mesure utilisés correspondent à l'étape finale du circuit. Les déchets sont souvent mélangés et les centres de traitements desservent des zones géographiques plus importantes que les périmètres communaux. L'imprécision de la classification des déchets engendre des confusions lors des pesées. Ainsi, une collecte 
traditionnelle pesée entrera dans la catégorie déchets ménagers alors qu'elle contient une part non définie de DNM.

Figure 4. : Mode de gestion des déchets ménagers et assimilés

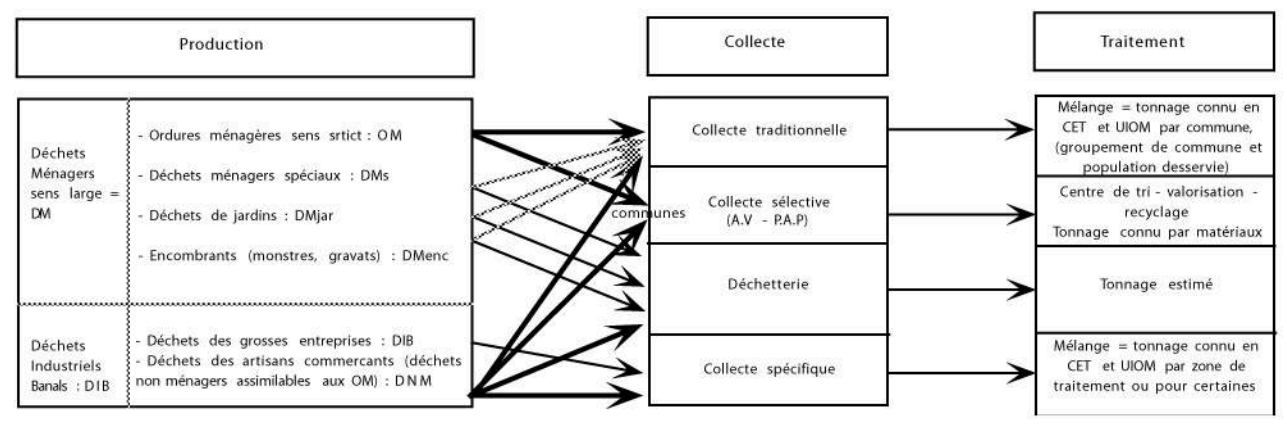

\section{B. Intérêt d'élaborer des indicateurs d'approche des besoins}

En France, la quantité de déchets est d'environ $1 \mathrm{~kg} / \mathrm{hab} / \mathrm{jour}$. Ce chiffre représente le résultat d'une simple opération: la production de déchets traités sur une zone géographique délimitée rapportée à la population desservie par cette zone de traitement. Il est utilisé comme référence dans l'organisation de la gestion des déchets municipaux. Pourtant, ce ratio ne représente qu'une moyenne nationale qui intègre des situations diverses et masque d'importantes variations locales : il peut être divisé par trois dans le monde rural ou multiplié par cinq et même plus à Paris (Rodier M., 1996). La figure 5 donne un exemple des tonnages obtenus par extrapolation pour la France, sachant que ce ne sont que des estimations générales.

Figure 5 : Types de déchets ménagers et assimilés

\begin{tabular}{|c|c|c|c|c|c|}
\hline $\begin{array}{l}\text { Déchets } \\
\text { de la } \\
\text { collectivités } \\
\text { locales }\end{array}$ & \multicolumn{3}{|c|}{$\begin{array}{c}\text { Déchets des ménages } \\
24,5, \mathrm{Mt}\end{array}$} & \multicolumn{2}{|c|}{$\begin{array}{c}\text { Déchets industriels } \\
\text { banals } \\
100 \mathrm{Mt}\end{array}$} \\
\hline \multirow{2}{*}{$\begin{array}{l}\text { Espaces verts } \\
\text { marchés, } \\
\text { nettoiement, } \\
\text { assainissement. } \\
22,5 \mathrm{Mt}\end{array}$} & \multirow{2}{*}{$\begin{array}{c}\text { Déchets } \\
\text { occasionnels } \\
\text { des ménages : } \\
\text { encombrants, } \\
\text { jardinage, } \\
\text { bricolage... } \\
4,5 \mathrm{Mt}\end{array}$} & \multicolumn{2}{|c|}{$\begin{array}{l}\text { Ordures ménagères (sens strict) } \\
\qquad 20 \mathrm{Mt}\end{array}$} & \multirow{2}{*}{$\begin{array}{l}\text { Déchets des } \\
\text { artisans, } \\
\text { commerçants } \\
\text { établissements } \\
\text { divers, } \\
\text { collectés avec } \\
\text { les ordures } \\
\text { ménagères } \\
5 \mathrm{Mt}\end{array}$} & \multirow{2}{*}{$\begin{array}{l}\text { Déchets } \\
\text { industriels } \\
\text { banals } \\
\text { collectés } \\
\text { séparément } \\
95 \mathrm{Mt}\end{array}$} \\
\hline & & $\begin{array}{c}\text { Fraction } \\
\text { collectée } \\
\text { sélectivement } \\
1,5 \mathrm{Mt}\end{array}$ & $\begin{array}{c}\text { Fraction } \\
\text { collectée } \\
\text { en mélange } \\
18,5 \mathrm{Mt}\end{array}$ & & \\
\hline & & \multicolumn{3}{|c|}{ Ordures ménagères (sens habituel) : $25 \mathrm{Mt}$} & \\
\hline \multicolumn{5}{|c|}{ Déchets municipaux : $52 \mathrm{Mt}$} & \\
\hline
\end{tabular}

Source données : ADEME 1993/1994.

L'élaboration de données de cadrage pour déterminer des politiques, des réglementations, des facturations selon le service rendu et le dimensionnement des équipements, nécessite de définir un ratio plus fiable et d'étudier ses variations en fonction des contextes locaux. En l'absence d'un tonnage connu, dès qu'une évaluation 
d'une quantité de déchets ménagers s'avère nécessaire, l'unique recours consiste à multiplier un ratio par la population correspondante :

- Dans le cadre de la mise en place des plans départementaux de collecte et de traitement des déchets ménagers et assimilés, il faut dimensionner les futurs centres de traitement en fonction de la masse de déchets à recevoir, qu'il s'agisse d'usine d'incinération ou de plates-formes de compostage.

31 - Pour facturer le coût du service lorsqu'une municipalité ou une entreprise se charge de la collecte, du transport et/ou du traitement des déchets et que les centres de traitements ne sont pas munis de pont-bascule pour procéder à des pesées.

32 - Pour le recouvrement de la taxe sur les décharges $(20 \mathrm{~F} / \mathrm{t}$ depuis le premier avril 1993), s'il n'existe aucun moyen de peser les déchets à enfouir.

33 - Pour évaluer la part d'ordures ménagères valorisables et choisir les filières de collecte sélective à mettre en place.

34 La politique engagée de modernisation de la gestion des déchets municipaux doit s'appuyer sur une connaissance systématique des ordures ménagères, afin de les valoriser au maximum dans les meilleures conditions. Des indicateurs de production spécifiques sont nécessaires pour effectuer des évaluations par commune.

\section{Propositions pour la détermination d'indicateurs de production spécifiques}

Sur un territoire communal, la quantité globale et la composition du gisement de déchets générés dépend de multiples facteurs: du niveau de vie, du mode de distribution des marchandises (commerce de proximité ou grande distribution), de l'urbanisme (taille des logements, densité du bâti), des catégories socioprofessionnelles, de la taille des ménages et notamment du nombre de commerçants et d'artisans pour les DNM. Les statistiques nationales montrent que les communes urbaines génèrent des quantités d'ordures ménagères supérieures à celles des communes rurales par habitant et par an. Le poids supérieur de déchets dans les grandes villes est-il seulement dû à la production des ménages? Si les communes ne génèrent effectivement pas les mêmes quantités et qualités d'ordures ménagères, il faut déjà savoir si cette disparité est due au comportement propre des ménages, en tant que principaux producteurs, avant de pouvoir affirmer qu'il existe des indicateurs d'émission propre liés aux spécificités des communes, autre que la population elle-même. En raisonnant à l'échelle communale, et pour la globalité du gisement des déchets municipaux, il apparaît évident que la composante structurante de l'organisation socio-économique et spatiale du territoire communal est un des critères pertinent et explicatif des variabilités des gisements des déchets collectés. Par contre, si on raisonne en terme de production d'ordures ménagères sans les DNM, l'échelle d'étude doit être fondée sur celle du ménage en tant que principal producteur d'ordures ménagères et non pas sur celle de la commune. Par conséquent, il faut savoir si cette différence de quantité d'ordures ménagères entre le milieu urbain et rural est due uniquement aux spécificités des communes (agricoles, touristiques, dortoirs, industrielles, urbaines...), qui peuvent alors mettre en évidence d'autres indicateurs de production spécifiques d'ordures ou, au contraire, si la production d'ordures ménagères est indépendante de cette spécificité. Dans ce cas, un facteur très influent ne serait-il pas la taille du ménage plutôt que le nombre 
d'habitants ? Donc, avant de créer des indicateurs de production spécifique, il faut déjà répondre aux problèmes de base suivants :

- Pourquoi les communes françaises ne génèrent-elles pas les mêmes quantités et qualités d'ordures ménagères par habitant et par an ? Pourquoi en milieu urbain, ces quantités sont-elles supérieures à celles du milieu rural ?

37 - Est-ce que les spécificités des communes ont une influence dans la production des ordures ménagères?

Une orientation envisagée consisterait à vérifier les hypothèses suivantes qui devraient permettre d'améliorer la connaissance concernant les ordures ménagères et de créer des indicateurs spécifiques de production, à savoir,

- La production des ordures ménagères par habitant et par an ne serait pas un facteur explicatif suffisant des variations observées par commune.

40 - Les modes de gestion de collecte des déchets ménagers et assimilés seraient responsables des variations observées des productions d'ordures ménagères par commune. En effet, ces variations résulteraient de l'adjonction des DNM dans la collecte traditionnelle.

\section{Conclusion}

41 La mise en place d'un nouveau système de gestion des ordures ménagères ne pourra s'avérer efficace que lorsque les problèmes de base auront été résolus. En l'absence de données précises sur les ordures ménagères produites par les ménages aucune analyse fiable n'est envisageable. Tant que les communes ne disposeront pas de ces éléments fondamentaux, elles ne pourront pas se diriger efficacement vers une gestion optimale - valorisation - et transparente - facturation du service rendu - de leurs déchets. De nouveaux procédés de mesure sont apparus dernièrement, tels l'ère de l'informatique embarquée de la collecte et du traitement des déchets. En munissant chaque conteneur d'une puce électronique, ces procédés permettent en temps réel, de connaître le taux de remplissage de la benne du camion et d'identifier le producteur. Leur utilisation sur un site teste convenablement choisi rendrait donc possible la validation de nos hypothèses. Le déchet urbain entre à son tour dans l'ère de la haute technicité de par les installations de collecte et de traitement, en étant équipé de compteurs à poubelle, comme pour l'eau et l'électricité.

\section{BIBLIOGRAPHIE}

ADEME (1993 (a)) - Collecte, traitement et stockage des déchets ménagers, État des techniques, Angers, $60 \mathrm{p}$.

ADEME (1993 (b)) - Incinération des résidus urbains : technique, réglementation, perspectives d'évolution, Angers, $44 \mathrm{p}$. 
ADEME (1993 (c)) - Plans départementaux d'élimination des déchets ménagers et assimilés. Méthodologie d'élaboration, Angers, $82 \mathrm{p}$.

ADEME (1994) - Les déchets en chiffres 1994, Angers, 146 p.

BERTOLINI G. (1990) - Le marché des ordures. Économie et gestion des déchets ménagers, L'harmattan, Paris, $206 \mathrm{p}$.

DEFEUILLEY C. (1996) - Le service public au défi de l'efficacité économique. Les constats de délégation dans la gestion des déchets ménagers. Thèse Sorbonne, Paris, 345 p.

LE BOZEC A. (1994) - Le service d'élimination des ordures ménagères (organisation, coûts, gestion), L'harmattan, Paris, $457 \mathrm{p}$.

PELCHAT M. (1993) - Rapport sur les problèmes posés par les déchets ménagers, Assemblée nationale, rapport $\mathrm{n}^{\circ} 3246$, enregistré le 18 janvier, Paris, $94 \mathrm{p}$.

RODIER M. (1996) - Ratios unitaires de production de déchets ménagers, Mémoire de DEA Gestion et traitement des déchets, Institut National des Sciences Appliquées de Lyon, 60 p.

\section{Bibliographie complémentaire}

ADEME (1993) - Mise en décharge : technique, réglementation, perspectives d'évolution, Angers, 29 p.

ADEME (1994) - Bilan et perspectives des plans départementaux d'élimination des déchets ménagers et assimilés, Angers, $8 \mathrm{p}$.

ADEME (1995) - Les déchets en France, Paris, 16 p.

ADEME (1996 (1)) - Déchets industriels banals : quels tonnage ? Rapport d'étude, 84 p.

ADEME (1996 (2)) - La taxe sur le traitement et le stockage des déchets et les aides financières correspondantes, Paris, $27 \mathrm{p}$.

ADEME (1997) - Gisement et valorisation des emballages en France, Données et références, Ademe, 11 p. BERTOLINI G. (1978) - Rebuts ou ressources ? la socio-économie du déchet, Éd entente, les cahiers de l'écologie, Paris, $155 \mathrm{p}$.

BERTOLINI G. (1995) - La double vie de l'emballage Economica, Paris, 112 p.

BERTOLINI G. (1996) - Déchet mode d'emploi Economica, Paris, 159 p.

GOUHIER J. (1997) - «Évolution historique de la gestion des déchets urbains en Occident », Annales des Mines, Paris, 11 p.

MAYSTRE L. Y. (1994) - Déchets urbains. Nature et caractérisation, Presses Polytechniques et Universitaires Romandes, Lausanne, 219 p.

\section{NOTES}

1. Les déchets urbains sont synonymes de déchets municipaux ou de résidus urbains. C'est l'ensemble des déchets dont l'élimination doit être assurée par les communes. Les déchets municipaux sont tous les déchets qui apparaissent sur le territoire communal, mais qui ne sont pas liés aux processus industriels de fabrication. Cette catégorie est très large et regroupe en fait plusieurs sous-catégories : les déchets ménagers (DM) ou déchets des ménages (comprenant les encombrants, ordures ménagères ( $\mathrm{OM})$, déchets de jardin,...), les déchets d'origine commerciale ou artisanale assimilables aux ordures ménagères et les déchets de voirie. 
2. Ordures ménagères : «Déchets de l'activité domestique quotidienne des ménages qui peuvent être pris en compte par la collecte traditionnelle » (ADEME). Il s'agit de ce qui est jeté chaque jour à la poubelle (restes de nourriture, verre, papier, carton, plastique, etc.).

3. Centre d'Enfouissement Technique

4. Groupe d'Étude pour l'Élimination des Résidus Solides

5. Agence National pour la Récupération et l'élimination des Déchets

6. Centre de Recherche des Études et Observations des Conditions de Vie

7. Agence de l'Environnement et de la Maîtrise de l'Énergie

8. Not In My Backyard : pas dans mon jardin, pas dans ma cour

9. Fond de Modernisation de la Gestion des Déchets

10. Déchets Non Ménagers en provenance des artisans et commerçants assimilables aux DM au sens de loi du 15 juillet 1975. Ils peuvent donc être collectés avec la collecte traditionnelle des O.M.

11. Usine d'Incinération des Ordures Ménagères

12. Déchets Industriels Banals: déchets de grosses entreprises assimilables aux ordures ménagères

13. Établissement Public de Coopération Intercommunale

\section{RÉSUMÉS}

Cet article traite des difficultés, pour les municipalités françaises, de la mise en place d'un nouveau système de gestion des déchets ménagers. La politique engagée de modernisation et d'efficacité en la matière, depuis la loi de 1992, implique une réorganisation complète des schémas traditionnels existants. Les municipalités doivent quitter une vision unitaire de l'élimination des déchets pour s'engager dans l'élaboration d'un système de gestion séparative et multiforme des ordures ménagères, afin de les valoriser au maximum dans les meilleures conditions. La question posée est de savoir si elles en ont actuellement les capacités?

This article concerns the difficulties for French Town Councils of settling up a new management system for collecting domestic rubbish. The modernization policy adopted to increase efficiency in this matter since the 1992 law, implies a complete reorganization of the whole traditional existing scheme. Town councils must give up an unitary outlook on rubbish disposal and commit themselves to developing a collection system which would allow rubbish to be classified in a multimodal way in order to maximise its value. The main question that remains is to know whether they currently have such a capability.

Dieser Artikel behandelt die Schwierigkeiten der französischen Gemeiden bei der Einführung eines neuen Systems für die Entsorgung von Haushaltmüll. Die seit dem Gesetz von 1992 engagierte Politik der Modernisierung und Effizienz schliesst eine vollständige Reorganisation der bestehenden traditionellen Schemata ein . Die Gemeinden müssen sich von der Vorstellung einer einheintlichen Müllensorgung trennen, um sich der Ausarbeitung eines Systems separativer und vielseitiger Entsorgung zu widmen, die den Müll maximal und unter besten Bedingung wiederverwertet. Hier wird die Frage gestellt, ob die Gemeinden dazu gegenwärtig die Kapazitäten haben. 
INDEX

Schlüsselwörter : Haushaltmüll, quantitative Einschätzung, Siedlungsabfälle

Keywords : domestic rubbish, municipal rubbish, quantitative evaluation

Mots-clés : déchets ménagers, déchets municipaux, déchets non ménagers, évaluation quantitative, ordures ménagères

\section{AUTEUR}

\section{ESTELLE KAH}

UFR de Géographie - Université Louis-Pasteur - 3, rue de l'Argonne 67000 Strasbourg 